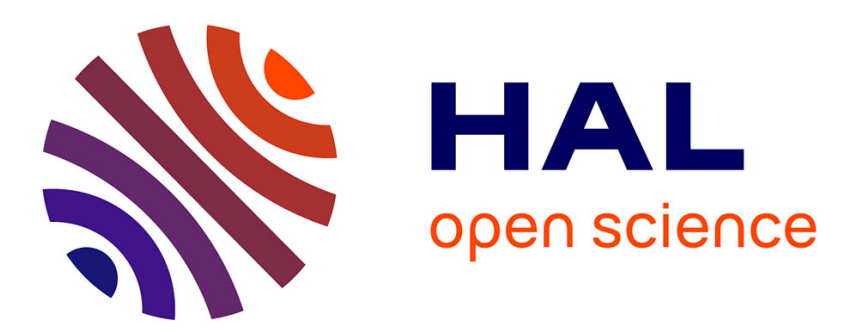

\title{
A saccadic choice task for target face detection at large visual eccentricities in patients with glaucoma
}

Boucart Muriel, Bachet Valentin, Corveleyn Xavier, Bacchetti Pascal, Jean Rouland, Muriel Boucart

\section{- To cite this version:}

Boucart Muriel, Bachet Valentin, Corveleyn Xavier, Bacchetti Pascal, Jean Rouland, et al.. A saccadic choice task for target face detection at large visual eccentricities in patients with glaucoma. Journal of Glaucoma, 2020, 97, pp.871-878. 10.1097/OPX.0000000000001586 hal-03018189

\author{
HAL Id: hal-03018189 \\ https://hal.science/hal-03018189
}

Submitted on 22 Nov 2020

HAL is a multi-disciplinary open access archive for the deposit and dissemination of scientific research documents, whether they are published or not. The documents may come from teaching and research institutions in France or abroad, or from public or private research centers.
L'archive ouverte pluridisciplinaire HAL, est destinée au dépôt et à la diffusion de documents scientifiques de niveau recherche, publiés ou non, émanant des établissements d'enseignement et de recherche français ou étrangers, des laboratoires publics ou privés. 
Boucart Muriel 1

Bachet Valentin 1-2

Corveleyn Xavier 3-4

Bacchetti Pascal 1-2

Rouland Jean Francois 1-2

\section{A saccadic choice task for target face detection at large visual eccentricities} in patients with glaucoma

1. University of Lille, INSERM, CHU Lille, UMR-S 1172 - Lille Neuroscience \& Cognition, F-59000 Lille, France

2. Lille university hospital, Hôpital Huriez, department of ophthalmology, Lille, France

3. University Côte d'Azur, LAPCOS, France

4. MSHS Sud-Est - Maison des Sciences de l'Homme et de la Société SudEst

Corresponding author: Muriel Boucart, Faculté de médecine de Lille, Pole Recherche, 1 place de Verdun, 59000 Lille, France. E-mail: muriel.boucart@chrulille.fr

Disclosure of funding: the authors received no funding for this study

Key words: glaucoma, scene perception, eccentricity,

\section{Word count:}

Abstract: 250

Manuscript: 3885

Figures: 5 
Abstract

Significance: Little is known about the perception of glaucomatous patients at large visual eccentricities. We show that the patients' performance drops beyond $40^{\circ}$ eccentricity even for large images of scenes suggesting that clinical tests should assess the patients' vision at larger eccentricities than 24 or $30^{\circ}$.

Purpose: Daily activities such as visual search, spatial navigation and hazard detection require rapid scene recognition on a wide field of view. We examined whether participants with visual field loss at standard automated perimetry 30-2 were able to detect target faces at large visual eccentricities.

Methods: Twelve patients with glaucoma and 14 controls were asked to detect a face in a two-alternative saccadic forced choice task. Pairs of scenes, one containing a face, were randomly displayed at $10,20,40,60$ or $80^{\circ}$ eccentricity on a panoramic screen covering $180^{\circ}$ horizontally. Participants were asked to detect and to saccade towards the scene containing a face.

Results: Saccade latencies were significantly slower in patients (264ms, CI: 222 to 306ms) than in controls (207ms CI: 190 to $226 \mathrm{~ms}$ ) and accuracy was significantly lower in patients (70\% CI: $75 \%$ to $85 \%)$ than in controls $(75.7 \%$ CI : $71.5 \%$ to $79.5 \%)$. Though still significantly above chance at $60^{\circ}$, the patients' performance dropped beyond $40^{\circ}$ eccentricity. The controls' performance was still above chance at $80^{\circ}$ eccentricity.

Conclusions: In patients with various degrees of peripheral visual field defect, performance dropped beyond $40^{\circ}$ eccentricity for large images at a high contrast. This result could reflect reduced spread of exploration in glaucoma. 
Glaucoma is an optic neuropathy characterized by structural (retinal ganglion cells and optic nerve fiber loss) and functional (visual field loss) deficit. The progressive loss of retinal ganglion cells is accompanied by matching retinotopic loss of contrast sensitivity in the visual field. Loss of contrast sensitivity due to glaucoma starts in peripheral vision but anatomical, ${ }^{1}$ physiological ${ }^{2}$ and behavioral data ${ }^{3-5}$ indicate that central vision is also affected. Few experimental studies have investigated glaucomatous patients' performance at very large visual eccentricities (above $50^{\circ}$ ). However, most of the visual field is in peripheral vision, regardless of how peripheral vision is defined (i.e., starting after the fovea, the perifovea or the macula). ${ }^{6}$ Detecting an object at large visual eccentricity is frequent in daily life. Indeed, the detection of relevant stimuli like a pedestrian crossing, a moving car and a facial expression at peripheral locations might be critical in everyday life. Daily activities like visual search, spatial navigation (walking or driving), spatial memory and detection of potential danger require quick scene gist recognition on a wide field of view. Despite its low spatial resolution, peripheral vision provides critical information about the environment. Experimental studies in normally sighted participants have shown that the coarse information provided by peripheral preview guides eye movements towards targets in visual search tasks ${ }^{7}$ and peripheral vision is efficient for scene gist recognition at very large eccentricities (above $\left.60^{\circ}\right) .{ }^{6,8}$

As far as glaucoma is concerned, studies on perception in peripheral vision have been conducted with driving simulators or in laboratory-based hazard detection tasks on real-world driving scenes, mostly with relatively narrow fields of view or relatively small eccentricities, for the location of road hazards (around $30^{\circ}$ ), see Kasneci et al. for a review. ${ }^{9}$ For instance, some studies showed lower pedestrian detection rates in the patients' group than in the control individual variability was noted, detection rates varying from $27 \%$ to $90 \%$. Other studies showed delayed first fixation on peripheral hazards in patients compared to controls ${ }^{11}$ or a higher number of collisions in patients than in controls. However, this was observed only in older patients with lower acuity. ${ }^{12}$ Szlyk et al. ${ }^{13}$ used a wider field of view with three monitors providing a visual field of $160^{\circ}$ horizontal viewing. They reported that patients with glaucoma exhibited a higher rate of simulator accidents than controls, but the visual eccentricity of hazards was not reported. 
The present study was designed to assess visual perception at eccentricities larger than those classically measured by automatic standard perimetry and seldom explored in experimental studies. In glaucoma, peripheral fibers of the optic nerve are affected first suggesting that the signal transmitted to the part representing the peripheral visual field in the cortex might be degraded. To assess signal processing in peripheral vision in glaucoma, we used a panoramic screen covering $180^{\circ}$ of the visual field. In a two-alternative forced choice task, participants were asked to saccade towards the scene containing a human face, at eccentricities varying from 10 to $80^{\circ}$ on each side of central fixation. Human faces were used as targets as previous studies in normally sighted participants have demonstrated that human faces elicit the fastest saccadic responses, faster than other stimuli like animals or transports at small eccentricities $\left(8^{\circ}\right)^{14}$; and that the faster saccades for human faces extend across the whole visual field (up to $80^{\circ}$ in young participants). ${ }^{15}$ Based on clinical data, we could have expected performance to deteriorate in extrafoveal vision in patients with glaucoma. However, conventional automated perimetry measures performance at levels of luminance close to threshold, and for randomly distributed small visual spots. Several studies have shown that increasing the size of stimuli in conventional automated perimetry (e.g., from 0.01 to $2.67^{\circ}$ ) improves detection performance and reduces variability in participants with glaucoma. ${ }^{16,17}$ As our stimuli were wide and presented at a high contrast, we expected the target to be detected above chance at large eccentricities, even for patients showing reduced sensitivity in peripheral vision on the visual field test. In line with previous studies on eye movements in glaucoma, ${ }^{18,19}$ we expected longer saccade latencies for patients than for controls.

\section{$\underline{\text { Participants }}$}

Patients with glaucoma

Fourteen adults ( 8 females) ranging in age from 46 to 74 years, mean 62.7 years, with bilateral primary open angle glaucoma agreed to participate in the experiment. However, two patients were excluded owing to difficulties in calibration of the eye tracker. The patients 
were recruited in the ophthalmology department at Lille university hospital. They were being treated for glaucoma. To be included in the study, patients had to have a visual acuity of 0.1 LogMar or better in each eye, a score above 26/30 on the Mini-Mental State Examination ${ }^{20}$ for people above 60 years old indicating that they had no major cognitive deficits, no other ocular disease than glaucoma (except for uneventful lens replacement cataract surgery), no history of neurological disease and no medication affecting attention. All participants underwent SITA-standard 30-2 perimetry on a Humphrey Visual Field Analyzer (HFA, Carl Zeiss Meditec, CA, USA) showing glaucomatous defects that were consistent with structural damage, as observed in OCT and fundus. We did not use the 60-4 Humphrey visual field analyser as, to our knowledge, there are no normative data on this test.

\section{Normally sighted age-matched controls}

Fourteen normally sighted people ( 9 females) ranging in age from 45 to 74 years, mean 61.1 years, were included. They were either a relative or a friend of patients. Controls had a full eye examination to ensure that they had no ocular pathology. To be included in the study, controls had to have a visual acuity of $8 / 10$ or better in each eye on the Monoyer scale (i.e., 0.1 to 0.0 LogMar), a score above 26/30 on the Mini-Mental State Examination for people above 60 years old and no history of neurological or psychiatric disease.

Both patients and controls were tested binocularly. The study was approved by the ethics committee of the university. In accordance with the tenets of the Declaration of Helsinki, written informed consent was obtained from all participants.

The demographic details and clinical data of the 12 patients and 14 controls are summarized in Table 1. The patients' visual fields are presented in Figure 1. (1)

[Table 1 and Figure 1 about here]

\section{$\underline{\text { Stimuli }}$}


The stimuli consisted of 275 grey-level photographs of human faces from various ethnic groups and 400 photographs of various objects (animals, vehicles, plants, buildings) in scenes containing no human faces, selected from a large photo-library database (Corel). Examples of stimuli are presented in Figure 2. For all photographs, the resolution was 512 x 512 pixels, covering $18^{\circ} \mathrm{X} 18^{\circ}$ of visual angle at a viewing distance of $2.04 \mathrm{~m}$. As contrast sensitivity rapidly decreases at large eccentricities, especially for high spatial frequencies, ${ }^{21}$ the images were presented at their original full contrast.

\section{$\underline{\text { Apparatus }}$}

The stimuli were displayed by means of three projectors (Optoma HD83) fixed on the ceiling and connected to a PC computer (Dell). Participants were seated 2.04 meters from a hemispheric rigid light grey $(68 \mathrm{~cd} / \mathrm{m} 2)$ screen covering $90^{\circ}$ eccentricity on each side of central fixation (see Figure 2). The presentation software was written in Matlab (MathWorks, Inc., Natick, MA, USA) by the lab programmer. Saccade responses were recorded by means of the iViewXTM HED eye tracker from SensoMotoric Instruments (Teltow, Germany) with a scene camera. The video-based eye tracker is head-mounted, using infrared reflection to provide an eye-in-head signal at a sampling rate of $50 \mathrm{~Hz}$ and accuracy of about $1^{\circ}$. The scene camera mounted on the head was positioned so that its view field was aligned with the observer's line of sight. Calibration was performed using a five-point grid. Following calibration, the eye tracker creates a cursor indicating an eye-in-head position that is merged with the video from the scene camera. The video records were analyzed using the software BeGaze from SensoMotoric Instruments (Teltow, Germany). We recorded the latency of the first saccade (from the onset of the photographs). As the scene camera covers $40^{\circ}$ and the hemispheric screen covers $180^{\circ}$, we could only record the direction of the saccade (left/right), not its amplitude. Before the experiment, participants were presented with a central white square $\left(40^{\circ} \mathrm{X} 40^{\circ}\right)$ containing five calibration points. They were asked to fixate the black dots (center, top right, top left, bottom right, bottom left) while their eye positions were recorded by the system. Once the calibration was completed, this was removed and participants started the saccadic-choice task. As the iViewXTM HED head-mounted eye tracker records eye movements only on one eye, half of the participants in each group were recorded on the left eye and the other half on the right eye. 


\section{Procedure}

The experimental trials were organized as follows: a black fixation cross $\left(5^{\circ} \mathrm{X} 5^{\circ}\right)$ was displayed centrally for $1 \mathrm{~s}$. It was followed by a gap (blank screen) of $200 \mathrm{~ms}$. A gap task is known to facilitate the production of express saccades. ${ }^{22}$ Following the gap, a pair of photographs (a target and a distractor) were simultaneously presented left and right of fixation, at the same eccentricity, for $1 \mathrm{~s}$. The inter-trial interval was fixed at $1500 \mathrm{~ms}$. Human faces were defined as targets. There were 160 trials determined by 5 eccentricities $(10,20,40$, 60 and $80^{\circ}$ ) X 2 spatial locations of the target (left/right of fixation) X 16 images randomly software-selected within the folders of 275 photographs of human faces and 400 photographs of objects. Images were presented only once. The 5 eccentricities and the 2 spatial locations of the target were randomly and equally represented. Participants were asked to saccade towards the scene containing a human face. A saccadic forced choice task was preferred to a manual (left/right) forced choice task because previous studies with other populations of patients (Alzheimer disease) have shown that saccade responses were more sensitive to show deficits than a manual response task in which patients have time to explore images before responding. ${ }^{23}$ We measured accuracy in target detection. As the scene camera of the eye tracker did not cover the whole screen accuracy was determined by the direction of the saccade.

[Figure 2 about here]

\section{Data analysis}

An ANOVA was conducted on the data (saccade latencies and accuracy) using the IBM SPSS Statistics 20 software. Whenever Mauchly's test ${ }^{24}$ indicated that the assumption of sphericity had not been fulfilled, the degrees of freedom were adjusted using the Greenhouse-Geisser correction. For pairwise comparisons, degrees of freedom were also corrected whenever the assumption of homogeneity of variance had been violated. The within-subject factors were the spatial location of the target (left/right) and eccentricity $\left(10^{\circ}, 20^{\circ}, 40^{\circ}, 60^{\circ}\right.$ and $\left.80^{\circ}\right)$. Group (patients/controls) was a between-subject factor. The mean saccade latencies and accuracy are presented in Figures 3 and 4. Individual data for patients and controls are presented in Figure 5 for accuracy. 


\section{RESULTS}

There was no significant main effect of the spatial location (left/right) of the $\operatorname{target}\left(F_{1,24}=\right.$ $.003, P=.955$ for saccade latencies and $F_{1,24}=3.5, P<.070, \eta_{p}^{2}=0.13$ for accuracy). The results presented in Figure 3 and 4 are averaged over the spatial location of the target. As expected, accuracy decreased $\left(F_{4,96}=24.9, P<.001, \eta_{p}^{2}=0.509\right)$ and saccade latencies increased $\left(F_{4,96}=65.7, P<.001, \eta_{p}^{2}=0.732\right)$ as eccentricity increased. There was a significant group effect both on saccade latencies $\left(F_{1,24}=8.05, P<.009, \eta_{p}^{2}=0.251\right)$, with longer saccades for patients than for controls, and on accuracy $\left(F_{1,24}=5.28, P<.031, \eta_{p}^{2}=\right.$ 0.180 ), with a lower accuracy for patients than for controls.

Group interacted significantly with eccentricity on accuracy $\left(F_{3.24,77.8}=4.31, P<.003, \eta^{2}{ }_{p}=\right.$ 0.152). A separate analysis for each eccentricity showed that accuracy was better for controls than for patients at all eccentricities (see Figure 4), but the difference was significant only at large eccentricities (by $3.1 \%$ at $10^{\circ}: t_{14.45}=0.864, P=0.42$; by $2.9 \%$ at $20^{\circ}: t_{24}=0.722, P=$ 0.477 , by $1.4 \%$ at $40^{\circ}: t_{24}=0.281, P=0.781$, by $17.4 \%$ at $60^{\circ}: t_{24}=3.09, P<.005$; by $14 \%$ at $\left.80^{\circ}: t_{24}=2.71, P<.012\right)$. We checked whether accuracy was above chance $(50 \%)$ at the largest eccentricities. Performance was still significantly above chance for controls at $80^{\circ}$ $\left(66.7 \% t_{13}=4.3, P<.001\right)$. Accuracy was significantly above chance at $60^{\circ}$ eccentricity for patients $\left(60.35 \% t_{11}=2.3, P<.041\right)$, but it was not the case at $80^{\circ}\left(52.7 \% t_{11}=0.87\right.$, ns $)$. No interaction involving group reached statistical significance for saccades.

We checked for correlations (Spearman) between the mean deviation of the best eye and performance as well as between age and performance. As the visual field was tested monocularly and the saccadic choice task was performed binocularly, we presumed that the better eye would determine binocular sensitivity. It has been reported that the MD of the better eye correlated better with quality-of-life measures than the MD of the worse eye. ${ }^{25}$ 
eccentricity $(r=0.496), 20^{\circ}(r=0.322), 40^{\circ}(r=0.252), 60^{\circ}(r=0.126)$ and $80^{\circ}(r=0.154)$, and no significant correlation between the MD of the best eye and saccade latency at $10^{\circ}$ eccentricity $(r=0.287), 20^{\circ}(r=0.077), 40^{\circ}(r=0.336), 60^{\circ}(r=0.343)$ and $80^{\circ}(r=0.182)$. For patients, age was not significantly correlated with accuracy, but it was for saccade latency at eccentricities $40^{\circ}(r=0.516, P<.05), 60^{\circ}(r=0.593, P<.05)$ and $80^{\circ}(r=0.537, P<.05)$. Older patients exhibited longer saccade latencies as eccentricity increased. For controls, no significant correlation was observed either between age and saccade latency or between age and accuracy at each eccentricity.

\section{DISCUSSION}

Standard automatic perimetry is a useful clinical tool to assess deficit in contrast sensitivity due to glaucoma and to monitor the evolution of the pathology. However, it does not provide a complete picture of the patients' vision of the environment. Mönter et al. ${ }^{26}$ have developed a new technique of repeated kinetic presentations to estimate isopter positions without interactive input from the clinician, as it is the case in the manual kinetic Goldmann perimetry, to examine the peripheral field of patients with moderately advanced glaucoma. They found that patients with similar central visual field loss at the automated visual field test may have strikingly different peripheral visual field. This study shows that tests that examine the peripheral visual field beyond the range of static perimetry may provide relevant information both for clinicians and for patients (e.g., on their ability to quickly detect a peripheral signal). Indeed, Crabb et al. ${ }^{27}$ showed that patients are unaware of the true extent of their visual defect. At eccentricities in the range of those measured by automated perimetry peripheral visual field loss in glaucoma has little effect on the accomplishment of natural actions. ${ }^{28}$ Rubinstein et al. ${ }^{29}$ showed that visual localization and pointing precision to high contrast stimuli remained largely unaffected by reductions in visual field sensitivity within the central $15^{\circ}$ of vision in patients with glaucoma. In a driving simulator using a wider view field ( $160^{\circ}$ of horizontal viewing field), Szlyk et al. ${ }^{13}$ observed only 7 simulator accidents in 40 patients with peripheral visual field loss and 1 simulator accident in the control group $(\mathrm{N}=17)$, but the eccentricity and the size at which the road hazards were presented was not specified. Our results show that, when asked to detect a scene containing a human face on the $180^{\circ}$ of the visual field, the patients' performance is globally lower than that of controls, both in terms of accuracy and saccade latencies, but the difference is significant only beyond $40^{\circ}$ 
eccentricity. Except for patients 2 and 12, accuracy dropped at $40^{\circ}$ eccentricity, even though the scenes were displayed at a high contrast and covered $18^{\circ} \times 18^{\circ}$ of visual angle. This result can be explained by the loss of optic nerve fibers conveying information from the peripheral field of view. Another possible, and complementary, account is a reduced spread of exploration, reported in several studies in patients with glaucoma. ${ }^{30,31}$. Smith et al. ${ }^{30}$ showed that the average elliptical region scanned by glaucomatous patients, measured by a bivariate contour ellipse area (BCEA) analysis, was more restricted than that of controls (by 23\%) in free viewing of images of natural scenes subtending $20.3^{\circ} \mathrm{X} 14.9^{\circ}$. Glen et al. ${ }^{32}$ found that making larger saccades appeared to be associated with better face recognition performance for patients with bilateral visual field defects in their central $10^{\circ}$ of visual field. Wiecek et al. ${ }^{33}$ reported that fixation duration, saccade size, and number of saccades per trial were not significantly different between patients with peripheral field loss and normally sighted controls in a visual search task but they observed different strategies. Some patients made fewer eye movements into areas of impaired vision whilst others were more likely to make an eye movement into an unsighted location suggesting that some patients do not compensate for peripheral visual field loss. Studies on glaucoma often report large inter individual variability in eye movements, either in free viewing or in active viewing tasks, and large variability in compensatory strategies that are adopted by patients. ${ }^{9,30,32}$

The drop in accuracy at $40^{\circ}$ eccentricity might also reflect cortical remapping consecutive to reduced neural input to the visual cortex as a result of degeneration of retinal ganglion cells in glaucoma. Murphy et al. ${ }^{34}$ showed evidence that glaucoma deterioration is already present in the brain before substantial vision loss can be detected by clinical assessment. In a brain imaging study, Zhou et al. ${ }^{35}$ investigated changes in brain function corresponding to central $(< \pm 12$ degrees) and peripheral ( $> \pm 12$ degrees) vision in order to assess cortical remapping in glaucoma. The stimuli, covering $55^{\circ} \times 55^{\circ}$, were expanding rings and rotating wedges. They found that bold responses were similar in patients and controls in the central visual field but that they were attenuated in the peripheral visual field, in V1, V2 and V3, in patients with mild-to-moderate glaucoma compared to controls. Our behavioral data also show a substantial deficit in peripheral vision but only at large visual eccentricities. The difference between patients and controls was not significant below $40^{\circ}$. Though the fusiform face area (FFA), selective to faces, is located in the temporal cortex ${ }^{36}$ the processing of faces starts with the filters of area V1. ${ }^{37}$ A low quality signal send to FFA from the peripheral part of V1 could 
account for the failure to discriminate a target face from a distractor beyond $40^{\circ}$ eccentricity in patients.

Saccades were slower in patients than in controls. Using a novel methodology Crabb et al. ${ }^{38}$ demonstrated that, in addition to clinical measurements (visual field, intra-ocular pressure, $\mathrm{OCT}$ ), features extracted from extensive maps of saccades made during free viewing of TV films for several seconds could discriminate a group of patients with glaucoma from age-related normally sighted controls with a sensitivity of $76 \%$ by using the saccade maps alone. Evidence for impaired saccadic eye movements in glaucoma has also been reported in several other studies. For instance, Kanjee et al. ${ }^{18}$ had 16 patients and 21 controls saccade towards high-contrast red discs subtending $0.1^{\circ}$ in diameter, located $10^{\circ}$ left and right of central fixation. They found that median saccade response time was significantly increased (by $28 \mathrm{~ms}$ ) in patients with glaucoma compared to controls. They also reported that the duration, amplitude and peak velocity of saccades was not altered, suggesting that the glaucomatous pathological process seems to alter mainly the initiation of saccades. Lamirel et al. ${ }^{19}$ also reported delayed saccade latency, by $23 \mathrm{~ms}$ on average, during saccades towards a static target at $7^{\circ}$ eccentricity, in patients with glaucoma compared to controls. At larger eccentricities, Mazumdar et al. ${ }^{39}$ recorded eye movements towards target spots at 54 spatial locations, corresponding to the locations of the 24-2 Humphrey field analyzer. Glaucomatous eyes exhibited longer saccade response times (by 170 to $260 \mathrm{~ms}$ depending on age group) than normally sighted age-matched controls. Our results also show delayed saccade latencies in patients compared to controls, with an increase in the difference as eccentricity increases (by $36 \mathrm{~ms}$ at $10^{\circ}$ to $74 \mathrm{~ms}$ at $60^{\circ}$ ), and a lower difference at $80^{\circ}$, where patients were at chance.

With the same saccadic choice task as that used in the present study, but at $8^{\circ}$ eccentricity in normally sighted young participants, Crouzet and Thorpe ${ }^{14}$ observed that the fastest saccades were triggered for faces as early as $100-110 \mathrm{~ms}$ after stimulus onset. They suggested that, in addition to higher-order cortical structures involved in face perception in the temporal cortex, the processing required to initiate these fast responses might involve subcortical processing pathways such as the amygdala and the superior colliculus (SC). The $\mathrm{SC}$ is a pivotal structure in the regulation of eye movements in primates. ${ }^{40}$ It receives early visual input via the retino-tectal and retino-geniculo-cortico-tectal pathways ${ }^{41}$ The motor burst of collicular neurons serves as a signal for triggering both express and regular saccades ${ }^{22}$. Compared to age-matched controls Najjar et al. ${ }^{42}$ reported slower and hypometric saccades in patients with glaucoma at a pre-perimetric stage with no detectable glaucomatous visual 
field loss on standard automated perimetry. They suggested that these disruptions could originate from impaired neuronal signaling due to potential disordered cortical and subcortical saccadic regulation, particularly in the SC, as a result of neurodegeneration. Zhang et al. ${ }^{43}$ found significantly reduced fMRI responses to transient achromatic stimuli in the magnocellular layers of the lateral geniculate nucleus and in the superficial layer of the SC, as well as from the early visual cortices (V1, V2 and MT), in patients with early-stage glaucoma compared to controls. The delayed saccades in patients with glaucoma in the present study might reflect a slower connectivity and/or a slower image analysis before saccade initiation due to reduced contrast sensitivity. In monkeys, modulation of stimulus luminance and contrast has been shown to affect the timing and magnitude of visual responses in the SC 44,45 and in $\mathrm{V} 1.46$

Conclusion and limitations: The results of the present study are consistent with previous studies showing impaired eye movements in glaucoma, specifically for large eccentricities. There are several limitations in this study. The number of participants was small, and they exhibited various stages of visual field defect. A future study could include groups at different stages of the disease. Colored images might have captured attention better than achromatic images. A measure of head movements might have shown that patients move their heads more than normally sighted observers to compensate for visual field loss in peripheral vision. Nevertheless, such a simple test may provide a complement of the clinical assessment of patients. It can be used to complement information obtained with static perimetry in which a small spot at contrast threshold can easily be missed.

\section{References}

1. Hood DC. Improving our Understanding, and Detection, of Glaucomatous Damage: An Approach Based Upon Optical Coherence Tomography (OCT). Prog Retin Eye Res 2017;57: 46-75.

2. Jung KI, Jeon S, Kim YC, et al. Comparison of Pattern Electroretinograms of Glaucoma Patients with Parafoveal Scotoma versus Peripheral Nasal Step. Sci Rep 2019; 9: 3547. 
3. Lenoble Q, Lek JJ, McKendrick AM. Visual Object Categorisation in People with Glaucoma. Br J Ophthalmol 2016;100:1585-90.

4. Roux-Sibilon, A, Rutgé, F, Aptel, F, et al. Scene and Human Face Recognition in the Central Vision of Patients with Glaucoma. PLoS One. 2018; 13: e0193465.

5. Schafer A, Rouland JF, Peyrin C et al. Glaucoma Affects Viewing Distance for Recognition of Sex and Facial Expression. Invest Ophthalmol Vis Sci 2018 ;59:4921-28.

6. Loschky LC, Szaffarczyk S, Beugnet C, et al. The Contributions Of Central And Peripheral Vision to Scene-Gist Recognition with a $180^{\circ}$ Visual Field. J Vis. 2019; 19:15. doi: 10.1167/19.5.15.

7. Torralba A, Oliva A, Castelhano MS, et al. Contextual Guidance of Eye Movements and Attention in Real-World Scenes: The Role of Global Features in Object Search. Psychol Rev 2006.113:766-86.

8. Boucart $M$, Moroni $C_{2}$, Thibaut $M$, et al. Scene Categorization at Large Visual Eccentricities. Vis Res. 2013;86:35-42.

9. Kasneci E, Black AA, Wood JM. Eye-Tracking as a Tool to Evaluate Functional Ability in Everyday Tasks in Glaucoma. J Ophthalmol 2017:6425913.

10. Qiu C, Jung JH, Tuccar-Burak M, et al. Measuring Pedestrian Collision Detection With Peripheral Field Loss and the Impact of Peripheral Prisms. Transl Vis Sci Technol 2018;7:1. doi: 10.1167/tvst.7.5.1.

11. Lee SS, Black AA, Wood JM. Effect of Glaucoma on Eye Movement Patterns and Laboratory-Based Hazard Detection Ability. PLoS One 2017;12:e0178876.

12. Kunimatsu-Sanuki S, Iwase A, Araie M et al. The Role of Specific Visual Subfields in Collisions with Oncoming Cars during Simulated Driving in Patients with Advanced Glaucoma. Br J Ophthalmol 2017;101:896-901.

13. Szlyk JP, Mahler CL, Seiple W et al. Driving Performance of Glaucoma Patients Correlates with Peripheral Visual Field Loss. J Glaucoma. 2005;14:145-50.
Code de champ modifié Code de champ modifié Code de champ modifié

Code de champ modifié

Mis en forme : Français (France) 
14. Crouzet SM, Kirchner H, Thorpe SJ. Fast Saccades Toward Faces: Face Detection in Just 100 ms. J Vis 2010;10:16.1-17. doi: 10.1167/10.4.16.

15. Boucart M, Lenoble Q, Quettelart J, et al. Finding Faces, Animals and Cars in Far Peripheral V. J Vis 2016; doi: 10.1167/16.2.10.

16. Wall M, Kutzko KE, Chauhanf BC. Variability in Patients with Glaucomatous Visual Field Damage Is Reduced Using Size V Stimuli. Invest Ophthalmol Vis Sci 1997;38:426-35.

17. Redmond T, Garway-Heath DF, Zlatkova MB, et al. Sensitivity Loss in Early Glaucoma Can be Mapped to an Enlargement of the Area of Complete Spatial Summation. Invest Ophthalmol Vis Sci 2010;51:6540-48.

18. Kanjee R, Yücel YH, Steinbach MJ, et al. Delayed Saccadic Eye Movements in Glaucoma. Eye Brain 2012;4:63-8.

19. Lamirel C, Milea D, Cochereau I, et al. Impaired Saccadic Eye Movement in Primary Open-Angle Glaucoma. J Glaucoma 2014;23:23-32.

20. Folstein MF, FolsteinSE, McHugh PR. Mini-Mental State: A Practical Method for Grading the Cognitive State of Patients for the Clinician. J Psych Res 1975;12: 189-98.

21. Cannon MW Jr. Perceived Contrast in the Fovea and Periphery. J Opt Soc Am A 1985;2: 1760-8.

22. Sparks D, Rohrer WH, Zhang Y. The Role of the Superior Colliculus in SaccadeI: a Study of Express Saccades and the Gap Effect. Vis Res 2000;40:2763-77.

23. Boucart M, Calais G, Lenoble Q et al. Differential Processing of Natural Scenes in Typical and Atypical Alzheimer Disease Measured with a Saccade Choice Task. Front Integr Neurosci. 2014;60. doi: 10.3389

24. Mauchly JW. Significance Test for Sphericity of a Normal $n$-Variate Distribution. Annals of Mathematical Statistics 1940;11:204-9.

25. Gutierrez P, Wilson MR, Johnson CA, et al. The Influence of Glaucomatous Visual Field Loss and Health-Related Quality of Life. Arch Ophthalmol 1997;115:777-84. 
26. Mönter VM, Crabb DP, Artes PH. Reclaiming the Periphery: Automated Kinetic Perimetry for Measuring Peripheral Visual Fields in Patients with Glaucoma. Invest Ophthalmol Vis Sci 2017;58:868-75.

27. Crabb DP, Smith ND, Glen FC, et al. How Does Glaucoma Look? Patient Perception of Visual Field Loss. Ophthalmology 2013;120:1120-26.

28. Dive S, Rouland JF, Lenoble Q, et al. Impact of Peripheral Field Loss on the Execution of Natural Actions: A Study with Glaucomatous Patients and Normally Sighted People. J Glaucoma 2016;25:e889-96.

29. Rubinstein NJ, Anderson AJ, Ma-Wyatt A et al. The Effects of Ageing and Visual Field Loss on Pointing to Visual Targets. PLoS One 2014; 9: e97190.

30. Smith ND, Crabb DP, Glen FC et al. Eye Movements in Patients with Glaucoma When

Viewing Images of Everyday Scenes. Seeing and Perceiving 2012;25: 471-92

31. Asfaw DS, Jones PR, Mönter VM et al. Does Glaucoma Alter Eye Movements when Viewing Images of Natural Scenes? A between-Eye Study. Invest Ophthalmol Vis Sci 2018;59:3189-98.

32. Glen FC, Smith ND \& Crabb DP Saccadic Eye Movements and Face Recognition Performance in Patients with Central Glaucomatous Visual Field Defects. Vis Res 2013; $82: 42-51$

33. Wiecek E, Pasquale LR, Fiser J et al. Effects of Peripheral Visual Field Loss on Eye Movements During Visual Search. Front Psychol 2012;3:472. doi: 10.3389/fpsyg.2012.00472.

34. Murphy MC, Conner IP, Teng CY et al. Retinal Structures and Visual Cortex Activity are

Code de champ modifié Impaired Prior to Clinical Vision Loss in Glaucoma. Sci Rep 2016;6:31464. 
35. Zhou W, Muir ER, Kundandeep S et al. Retinotopic fMRI Reveals Visual Dysfunction and Functional Reorganization in the Visual Cortex of Mild to Moderate Glaucoma Patients. J Glaucoma 2017;26:430-7.

36. Kanwisher N, McDermott J, Chun MM. The Fusiform Face Area: a Module in Human Extrastriate Cortex Specialized for Face Perception. J Neurosci 1997;17:4302-11.

37. Keil MS. "I look in Your Eyes, Honey": Internal Face Features Induce Spatial Frequency Preference for Human Face Processing. PLoS Comput Biol 2009;5:e1000329.

38. Crabb DP, Smith ND, Zhu H. What's on TV? Detecting Age-Aelated Neurodegenerative Eye Disease Using Eye Movement scanpaths. Front Aging Neurosci 2014;6:312. doi: 10.3389

39. Mazumdar D, Pel JJ, Panday M et al. Comparison of Saccadic Reaction Time Between Normal and Glaucoma Using an Eye Movement Perimeter. Indian J Ophthalmol 2014;62: 559.

40. Shen K, Valero J, Day GS et al. Investigating the Role of the Superior Colliculus in Active Vision with the Visual Search Paradigm. Eur J Neurosci 2011;33:2003-16.

41. Cusick CG. Anatomical Organization of the Superior Colliculus in Monkeys: Corticotectal Pathways for Visual and Visuomotor Functions. Prog Brain Res 1988;75:1-15.

42. Najjar RP, Sharma S, Drouet M, et al. Disrupted Eye Movements in Preperimetric Primary Open-Angle Glaucoma. Invest Ophthalmol Vis Sci 2017;58:2430-37.

43. Zhang P, Wen W, Sun X, et al. Selective Reduction of FMRI Responses to Transient Achromatic Ttimuli in the Magnocellular Layers of the LGN and the Superficial Layer of the SC of Early Glaucoma Patients. Hum Brain Mapp 2016;37:558-69.

44. Marino RA, Levy R, Boehnke S, et al. Linking Visual Response Properties in the Superior Colliculus to Saccade Behavior. Eur J Neurosci 2012;35: 1738-52.

45. Marino RA, Levy R, Munoz DP. Linking Express Saccade Occurrence to Stimulus Properties and Sensorimotor Integration in the Superior Colliculus. J Neurophysiol 2015;114: 879-92.
Code de champ modifié 
490 46. Gawne TJ. The Simultaneous Coding of Orientation and Contrast in the Responses of V1

491 Complex Cells. Exp Brain Res 2000;133:293-302. 
495 Table 1. Demographic and clinical data of patients with glaucoma and normally sighted agematched controls. $\mathrm{LE}=$ left eye, $\mathrm{RE}=$ right eye, $\mathrm{MD}=$ mean deviation $. \mathrm{GCL}=$ Ganglion $\mathrm{Cell}$ measuring RNFL and GCL at the time of testing.

Fig.1. Patients' visual fields (Humphrey 30-2).

Fig.2. Example of display. Two scenes, one containing a target face, were projected simultaneously on a panoramic screen randomly at $10,20,40,60$ or $80^{\circ}$ eccentricity. A headmounted eye tracker recorded the direction of the first saccade towards the target face. with glaucoma (right panel). $\mathrm{X}=$ mean. Cercles = extreme values.

Fig.4. Boxplot of accuracy (\%) with standard deviations as a function of retinal eccentricities $\left(10\right.$ to $\left.80^{\circ}\right)$ in age-matched normally sighted controls (left panel) and in patients with glaucoma (right panel). $\mathrm{X}=$ mean. Cercles $=$ extreme values.

Fig.5. Individual data for 12 patients (right) and 14 controls (left) as a function of eccentricity. 


\begin{tabular}{|c|c|c|c|c|c|c|c|c|c|c|}
\hline \multirow{2}{*}{ Patients } & \multirow[t]{2}{*}{ Gender } & \multirow[t]{2}{*}{ Age } & \multirow{2}{*}{$\begin{array}{c}\text { Acuity } \\
\text { RE }\end{array}$} & \multirow{2}{*}{$\begin{array}{c}\text { (LogMAR) } \\
\text { LE }\end{array}$} & \multirow{2}{*}{$\begin{array}{c}\text { MD } \\
\text { RE }\end{array}$} & \multirow[b]{2}{*}{ LE } & \multirow{2}{*}{\begin{tabular}{|l} 
GCL \\
LE \\
\end{tabular}} & \multirow[b]{2}{*}{ RE } & \multicolumn{2}{|c|}{ RNFL } \\
\hline & & & & & & & & & LE & RE \\
\hline 1 & $\bar{F}$ & 63 & 0 & 0 & -12.15 & -16.17 & & & & \\
\hline 2 & $\mathrm{~F}$ & 67 & 0 & 0 & -6.67 & -7.98 & 73 & 74 & 76 & 78 \\
\hline 3 & $\mathrm{H}$ & 61 & 0.1 & 0 & -16.08 & -8.57 & 62 & 59 & 58 & 48 \\
\hline 4 & F & 70 & 0.1 & 0.1 & -6.02 & -10.93 & & & & \\
\hline 5 & $\mathrm{~F}$ & 74 & 0 & 0 & -22.14 & -20.74 & 52 & 33 & 61 & 68 \\
\hline 6 & $\mathrm{H}$ & 59 & 0.1 & 0 & -16.49 & -16.31 & 67 & 54 & 64 & 57 \\
\hline 7 & $\mathrm{H}$ & 49 & 0 & 0 & -12.9 & -11.13 & & & & \\
\hline 8 & $\mathrm{~F}$ & 63 & 0 & 0 & -21.68 & -22.47 & 64 & 73 & 59 & 63 \\
\hline 9 & $\mathrm{~F}$ & 74 & 0.1 & 0 & -14.48 & -9.11 & & & 63 & 58 \\
\hline 10 & $F$ & 58 & 0 & 0 & -16.2 & -11.2 & & & & \\
\hline 11 & $\mathrm{H}$ & 46 & 0 & 0 & -15.63 & -17.39 & 67 & 65 & 67 & 71 \\
\hline 12 & $\mathrm{~F}$ & 68 & 0 & 0 & -10.37 & -15.09 & & & & \\
\hline \multirow[t]{2}{*}{ Controls } & \multirow[t]{2}{*}{ Gender } & \multirow[t]{2}{*}{ Age } & \multicolumn{2}{|c|}{ Acuity (LogMAR) } & & & & & & \\
\hline & & & RE & LE & & & & & & \\
\hline 1 & $\bar{F}$ & 54 & 0 & 0 & & & & & & \\
\hline 2 & $\mathrm{H}$ & 74 & 0.1 & 0.1 & & & & & & \\
\hline 3 & $\mathrm{H}$ & 64 & 0 & 0 & & & & & & \\
\hline 4 & $\mathrm{~F}$ & 56 & 0 & 0 & & & & & & \\
\hline 5 & $\mathrm{H}$ & 57 & 0 & 0 & & & & & & \\
\hline 6 & $\mathrm{~F}$ & 66 & 0 & 0 & & & & & & \\
\hline 7 & $\mathrm{~F}$ & 76 & 0.1 & 0.1 & & & & & & \\
\hline 8 & $\mathrm{~F}$ & 60 & 0 & 0 & & & & & & \\
\hline 9 & $\mathrm{~F}$ & 45 & 0 & 0 & & & & & & \\
\hline 10 & $\mathrm{H}$ & 56 & 0 & 0 & & & & & & \\
\hline 11 & $\mathrm{~F}$ & 61 & 0 & 0 & & & & & & \\
\hline 12 & $\mathrm{~F}$ & 66 & 0.1 & 0.1 & & & & & & \\
\hline 13 & $\mathrm{H}$ & 61 & 0.1 & 0.1 & & & & & & \\
\hline 14 & $\mathrm{~F}$ & 59 & 0 & 0 & & & & & & \\
\hline
\end{tabular}


Figure 1

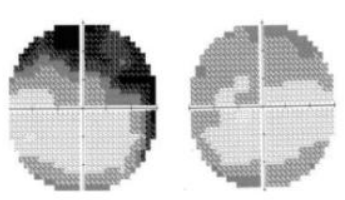

P1

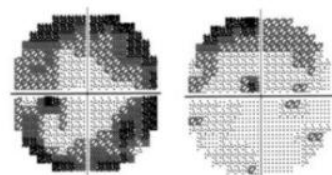

P4

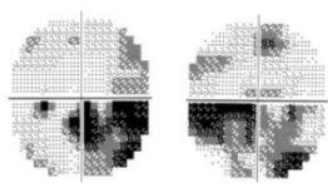

P7

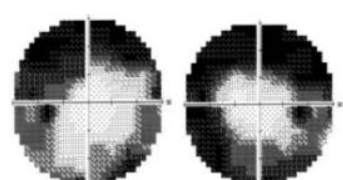

522

523

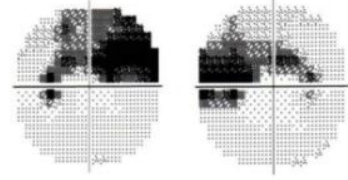

P2

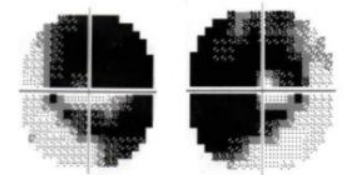

P5

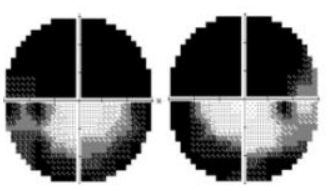

P8

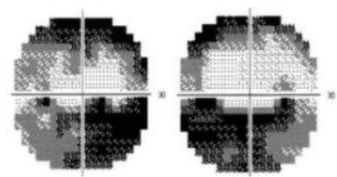

P11

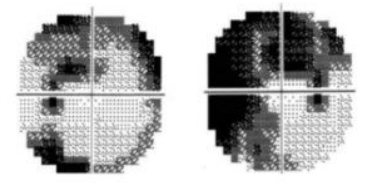

P3

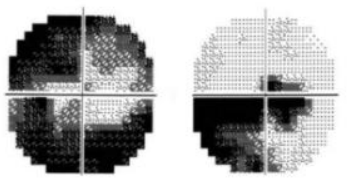

P6

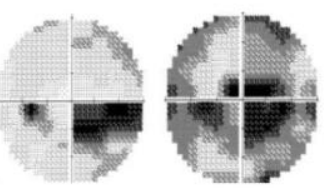

P9

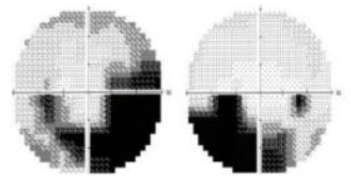

P12 
Figure 2

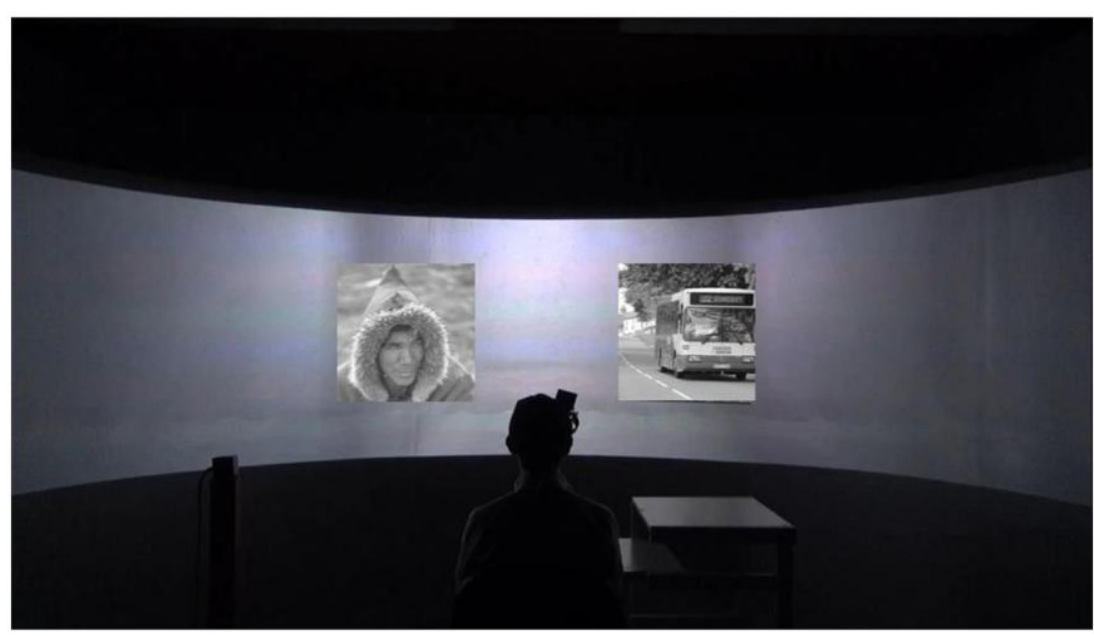

525

526 
527 Figure 3

528
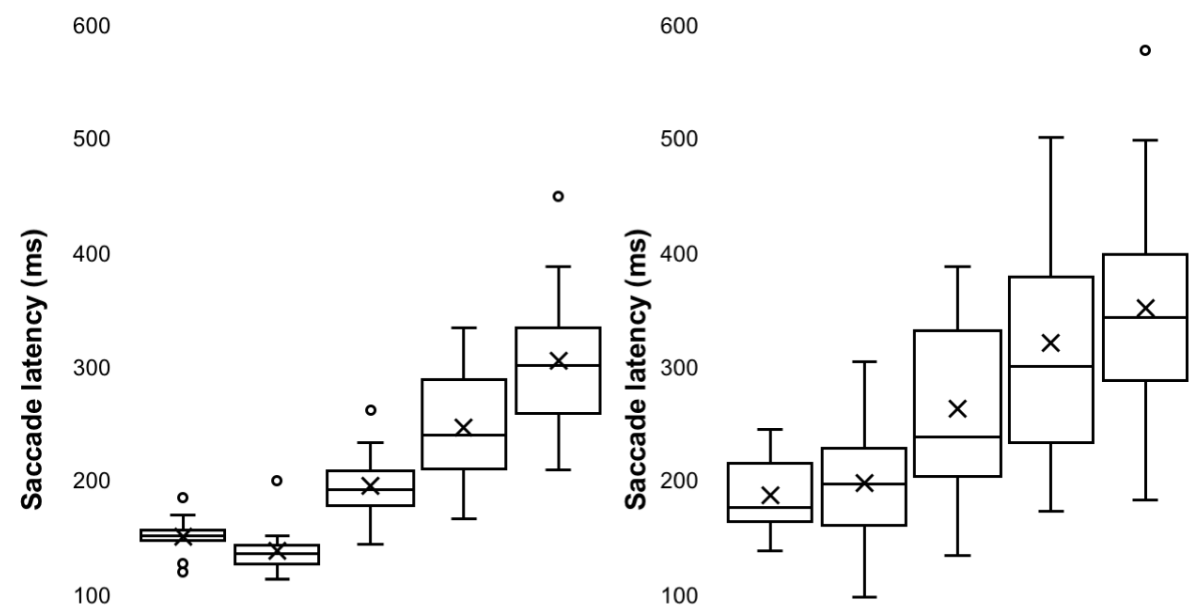

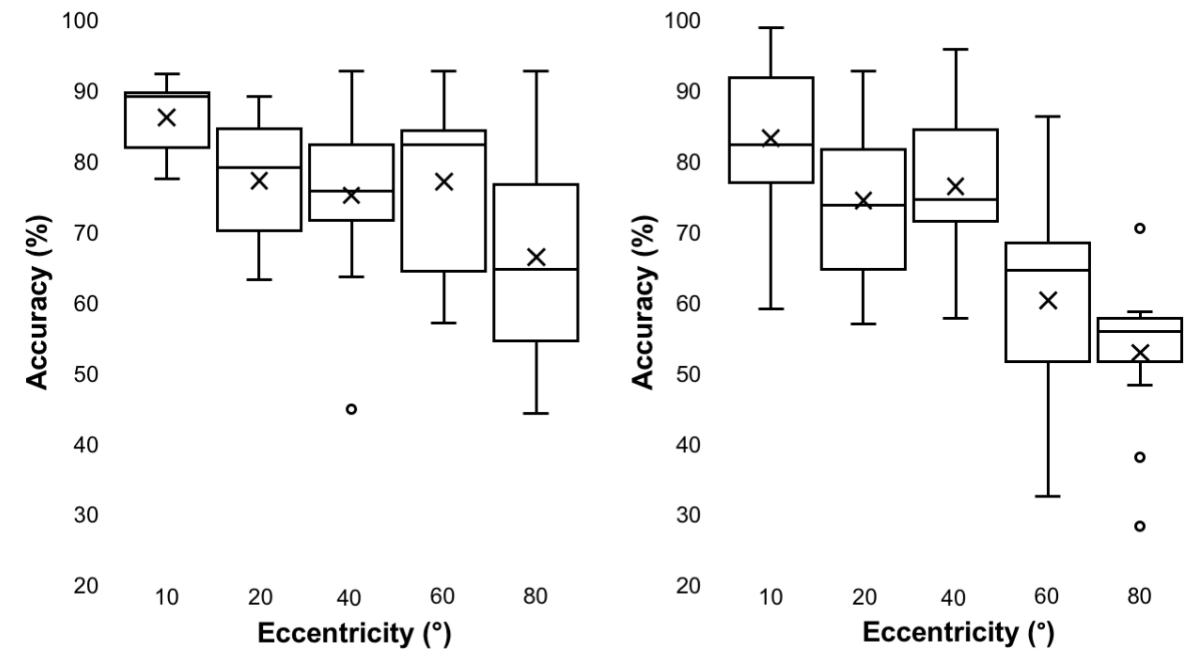

545

546 
Figure 5
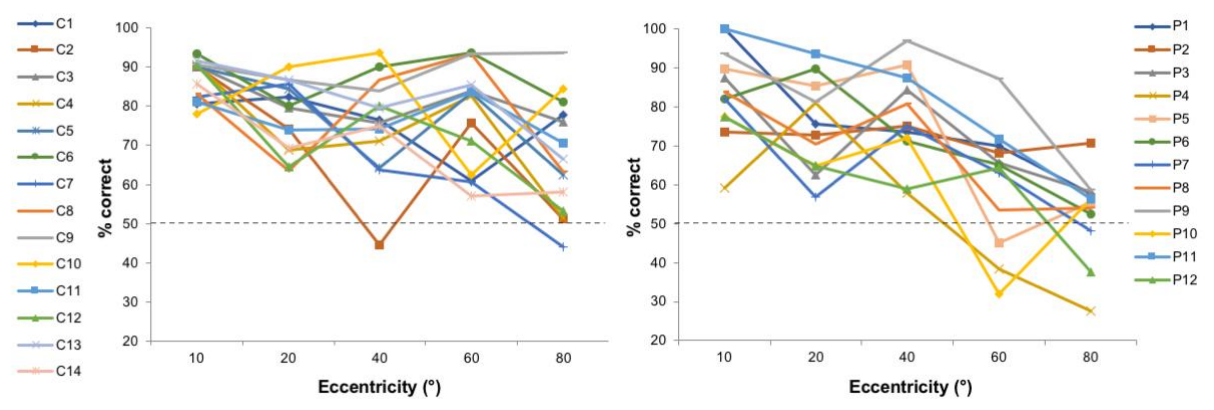